\title{
Malnutrition and Clinical Outcome in Urological Patients
}

\author{
A. Karl ${ }^{1}$, M. Staehler ${ }^{1}$, R. Bauer ${ }^{1}$, S. Tritschler ${ }^{1}$, Y. Hocaoglu 1 , A. Buchner ${ }^{1}$, J. Hoffmann ${ }^{2}$, D. Kuppinger ${ }^{2}$, \\ C. Stief ${ }^{1}$, P. Rittler ${ }^{2}$ \\ ${ }^{1}$ Department of Urology, University of Munich-Campus Grosshadern, Munich, Germany \\ ${ }^{2}$ Surgical Department, University of Munich-Campus Grosshadern, Munich, Germany
}

\begin{abstract}
Introduction: In a previous study we evaluated the risk for malnutrition among urological patients in a German university hospital. There are published different studies in other surgical fields that could show a correlation between malnutrition and clinical outcome. As data on this issue is still rare in the urological field we aimed to correlate the risk of malnutrition with different parameters regarding clinical outcome.

Methods: In the time from 2007 to 2009 a total of 320 patients were evaluated regarding the risk of malnutrition and occurrence of complications during the time of hospitalization at our Urological department. The Nutritional risk screening 2002 (NRS) by Kondrup et al. was used for the estimation of the risk level for malnutrition. Patients of a German university hospital were included independently of intervention, age or gender. Parameters for clinical outcome were: pulmonary complications (infectious/noninfectious), cardiovascular complications (infectious/noninfectious), other infections (urinary tract infection etc.), wound healing disorders and time of hospitalization.

Results: In this evaluation 320 patients were included for analysis. Forty patients $(13 \%)$ presented with a normal nutritional status (NRS score 0 ) at the time of admission to the hospital and 212 patients (66\%) were at risk for forming malnutrition problems (NRS score $1-2)$. Sixty eight patients $(21 \%)$ of this urological cohort were detected with a malnutrition according to the applied NRS Score $(\geq 3)$. Regarding the occurrence of overall complications in this cohort the rate was rather low compared to other surgical fields. Of 320 patients only 22 patients $(7 \%)$ presented with relevant complications during their hospitalization. However if data were stratified for peri- and postoperative complications in correlation to nutritional status of patients, an evident trend to a higher complication rate of $9 \%$ was obvious.

Conclusions: In our cohort of exclusively urological patients, the risk for post-surgical complications was higher in patients who were malnourished as defined using the Nutritional Risc Screening System (NRS) by Kondrup et al. Further studies need to show whether an adequate nutritional supportive therapy could help to optimize the clinical outcome of malnourished urological patients.
\end{abstract}

Key words: Malnutrition, Urology, Outcome, observational study

\section{INTRODUCTION}

The association between malnutrition and patients' outcome has been established for different diseases and medical fields. Large cohort studies could show a close association between malnutrition and increased complication rate, mortality, length of hospital stay and costs [2,9]. Hospital malnutrition in general has been investigated before, whereas no special interest was paid to urological patients in particular. As data on this issue seemed to be rare in the Urological field we performed a study in 2007 to estimate the prevalence of malnutrition among Urological patients on an operative ward of a German university hospital. Results of this study were published in Urology in 2009 [4]. According to this study, malnutrition was diagnosed in $16 \%$ of 897 included patients (NRS Score $\geq 3$ ). The NRS score was assessed at score 0 (no malnutrition), 1-2 (risk for malnutrition) and 3-5 (malnutrition) in 45, 708 and 144 patients, respectively. Age and malignant disease were significant risk factors for malnutrition $(p<0.001)$. Also the type of surgery was significantly associated with the risk of malnutrition $(p<0.001)$. Gender and body mass index had no significant influence.

As this former study focused exclusively on the estimation of the prevalence of malnutrition among urological patients the now presented follow up study aimed to detect a possible correlation between malnutrition and clinical outcome of urological patients.

As tool for the evaluation of malnutrition the $\mathrm{Nu}-$ tritional Screening Score by Kondrup at al. was used again.

\section{Methods}

In the time from 2007 to 2009 a total of 320 patients were screened and evaluated prospectively regarding the risk of malnutrition and the occurrence of complications during the time of hospitalization at our Urological department. The Nutritional risk screening 2002 (NRS) by Kondrup et al. was used for the estimation of the risk level for malnutrition of these patients. 
Nutritional Risk ScreEning (NRS 2002)

The screening tool used was based on the NRS 2002, provided by the Danish Society for Parenteral and Enteral Nutrition. A minimal amendment (categorization of different operative procedures) was created for use in urological patients. In brief, patients are characterized by scoring the components 'undernutrition' and 'severity of disease' in four categories (absent -0 pts., mild -1pt., moderate -2 pts. and severe -3 pts.). The patient can have a score of $0-3$ pts. for each component resulting in a total score of $0-6$ pts., plus 1 pt in case the patient is $>70 \mathrm{yrs}$ old. Any patient with a total score $>3$ is considered to be malnoursished. 'Undernutrition' is evaluated using three variables (BMI, percentage of recent weight loss and recent change in food intake). The most compromised of the three variables is used to categorize the patient. Detailed information about the development and validation of this tool is presented in the publication of Kondrup et al. [5].

Patients of a German university hospital were included independently of intervention, age or gender. Following parameters for clinical outcome were collected: pulmonary complications (infectious/noninfectious), cardiovascular complications (infectious/noninfectious), other infections (urinary tract infection etc.), wound healing disorders and length of hospital stay.

\section{RESULTS}

In this evaluation 320 patients were included for analysis. Forty patients $(13 \%)$ presented with a normal nutritional status (NRS score 0 ) at the time of admission to the hospital and 212 patients $(66 \%)$ were at risk for forming malnutrition problems (NRS score 1-2). Sixty eight patients $(21 \%)$ in this urological cohort were detected to be malnourished according to the NRS Score $(\geq 3)$. Regarding the occurrence of the overall complications in our cohort the rate was rather low com- pared to other surgical fields. Of 320 patients only 22 patients $(7 \%)$ presented with relevant complications during their hospitalisation.

If the occurrence of complications is stratified in relation to the nutritional status, a clear trend towards a higher complication rate among those patients who are at risk for malnutrition $(7 \%)$ or who are malnourished $(9 \%)$ can be found (Table 1).

If we take a closer look at the different kinds of complications (infectious or non-infectious) we could detect no significant difference between the patients that are well nourished. (3\%) However, similar to surgical patients, there can be detected a significant rise in complications related to infections in patients that are malnourished $(6 \%)$. In contrary to the results that can be found in pure surgical publications the group of patients with a relative risk for malnutrition had a higher rate of non-infectious complications. (1.8\% vs. $4.7 \%)$.

Further evaluation of the data was performed to classify for the reason of complications regarding pulmonary (e.g. embolia, pneumonia etc.) and cardiovascular complications (e.g. myocardial infarction, arythmia etc.) of non infectious and infectious origin (Table 2). Furthermore complications like wound healing disorders or general infections (urinary tract infections, intraabdominal abscess etc.) were analysed. Regarding the non-infectious pulmonary and cardiovascular complications the malnourished group showed a higher incidence than the patients without nutritional impairment.

Regarding the infectious pulmonary complications there was found a low incidence in the whole group of only $0.5 \%$.

Concerning the wound healing disorders there was detected a similar finding as found in surgical publications. Patients with a normal nutrition status had no disorders, whereas $0.5 \%$ of patients with the risk for malnutrition and $3 \%$ of malnourished patients were detected with wound healing disorders (Table 3).

Table 1. Patient characteristics.

\begin{tabular}{lcccc}
\hline & total & NRS Score 0 & Score 1-2 & Score $\geq 3$ \\
\hline Patients (n=320) & 320 & 40 & 212 & 68 \\
Percentage & $100 \%$ & $13 \%$ & $66 \%$ & $21 \%$ \\
No complications (n) & 299 & 38 & 199 & 62 \\
Percentage & $93 \%$ & $95 \%$ & $94 \%$ & $91 \%$ \\
Complications (n) & 22 & 2 & 14 & 6 \\
Percentage & $7 \%$ & $5 \%$ & $7 \%$ & $9 \%$ \\
\hline
\end{tabular}

Table 2. General complications (infectious / non-infectious) according the NRS Score.

\begin{tabular}{lccc}
\hline General Complications & Score 0 & Score $1-2$ & Score $\geq 3$ \\
\hline Infectious $=\mathrm{n}$ & 1 & 4 & 4 \\
$\%$ & $3.0 \%$ & $1.8 \%$ & $6.0 \%$ \\
Non-infectious $=\mathrm{n}$ & 1 & 10 & 2 \\
$\%$ & $3.0 \%$ & $4.7 \%$ & $3.0 \%$
\end{tabular}


Table 3. Specific complications.

\begin{tabular}{|c|c|c|c|}
\hline Specific Complications & Score 0 & Score 1-2 & Score $\geq 3$ \\
\hline $\begin{array}{l}\text { Pulmonary non infectious compl. } \\
\%\end{array}$ & $\begin{array}{c}0 \\
0.0 \%\end{array}$ & $\begin{array}{l}2 \\
1.0 \%\end{array}$ & $\begin{array}{c}0 \\
0.0 \%\end{array}$ \\
\hline $\begin{array}{l}\text { Myocardial comp. }=\mathrm{n} \\
\%\end{array}$ & $\begin{array}{c}0 \\
0.0 \%\end{array}$ & $\begin{array}{l}2 \\
1.0 \%\end{array}$ & $\begin{array}{c}1 \\
0.5 \%\end{array}$ \\
\hline $\begin{array}{l}\text { Pulmonary infectious complications }=\mathrm{n} \\
\mathrm{0}\end{array}$ & $\begin{array}{c}0 \\
0.0 \%\end{array}$ & $\begin{array}{l}1 \\
0.5 \%\end{array}$ & $\begin{array}{c}0 \\
0.0 \%\end{array}$ \\
\hline $\begin{array}{l}\text { Wound healing disorders }=\mathrm{n} \\
\%\end{array}$ & $\begin{array}{c}0 \\
0.0 \%\end{array}$ & $\begin{array}{l}1 \\
0.5 \%\end{array}$ & $\begin{array}{c}2 \\
3.0 \%\end{array}$ \\
\hline
\end{tabular}

Table 4. Mean time of hospitalisations.

\begin{tabular}{lccc}
\hline Mean time of hospitalisation & NRS Score 0 & NRS Score 1-2 & NRS Score $\geq \mathbf{3}$ \\
\hline No complications / hospital stay in days & 6 & 5 & 9 \\
Complications / hospital stay in days & 6 & 16 & 28 \\
\hline
\end{tabular}

\section{Discussion}

Our results show that not only surgical patients but also urological patients who are at risk for malnutrition suffer from a higher peri- and postoperative complication rate. Similar to patients published in surgical studies [1] we could detect a relation of other comorbidities and postoperative non-infectious complications. If we take a look at patients who were malnourished in our cohort, there could be detected a higher risk for infectious postoperative complications.

However in our urological cohort the situation differs from that published in surgical studies [3, 6-8]. In surgical patients there can be seen, that infectious and non-infectious complications are rising according to the state of malnutrition. The higher the risk for malnutrition, the higher was the risk for both infectious and non-infectious complications. However in our urological cohort of patients with malnutrition, the rate of infectious complications was lower than that of non-infectious complications. This shows that in this group of patients there were more cardiovascular and pulmonary comorbidities preexistent, which were responsible for the effect of complication-shift in the postoperative period.

Furthermore there remains the question for severity and value of complications, what can be estimated from the time of hospitalisation. For patients without important complications, the medial hospitalisation was 5-6 days, whereas patients with a relevant malnutrition presented with a medial hospitalisation time of 9 days. By looking at the patients with complications and their time of hospitalisation, there was seen no elongation of hospital stay in the group of normal nourished patients (NRS 0). However analyzing patients with the risk of malnutrition (NRS 1-2) there could be seen a significant elongation of hospitalisation up to 16 days. Analysing patients with existent malnutrition (NRS $\geq 3$ ) hospital stay was extended to 28 days (Table 4$)$.
From these results we can conclude, that malnourished patients experience more severe complications postoperatively or in case of complications with identical severity to other patients groups, show a significant worse convalescence.

\section{CONCLUSIONS}

In our cohort of exclusively urological patients, the rate of complications was higher in patients who were malnourished as defined using the Nutritional Risk Screening System (NRS) by Kondrup et al. Further studies need to address, if we can take influence on this issue by optimizing the preoperative nutrition status by special interventions like they were described in common and oncological surgery procedures. As we can find beneficial effects of the so called immunonutrition, it could be possible that these effects could be observed also in regular and oncological urological patients

\section{REFERENCES}

1. Bruun LI, Bosaeus I, Bergstad I et al.: Prevalence of malnutrition in surgical patients: evaluation of nutritional support and documentation. Clin Nutr 18(3): 141-147, 1999.

2. Correia MI and Waitzberg DL: The impact of malnutrition on morbidity, mortality, length of hospital stay and costs evaluated through a multivariate model analysis. Clin Nutr 22(3): 235-239, 2003.

3. Edington J, Boorman J, Durrant ER et al.: Prevalence of malnutrition on admission to four hospitals in England. The Malnutrition Prevalence Group. Clin Nutr 19(3): 191-195, 2000.

4. Karl A, Rittler P, Buchner A et al.: Prospective assessment of malnutrition in urologic patients. Urology 73(5): 1072-1076, 2009.

5. Kondrup J, Rasmussen HH, Hamberg O et al.: Nutritional risk screening (NRS 2002): a new method based on an analysis of controlled clinical trials. Clin Nutr 22(3): 321336, 2003. 
6. Kyle UG, Pirlich M, Schuetz T et al.: Prevalence of malnutrition in 1760 patients at hospital admission: a controlled population study of body composition. Clin Nutr 22(5): 473-481, 2003.

7. McWhirter JP and Pennington CR: Incidence and recognition of malnutrition in hospital. BMJ 308(6934): 945948, 1994.

8. Pirlich M, Schutz T, Norman K et al.: The German hospital malnutrition study. Clin Nutr 25(4): 563-572, 2006.

9. Waitzberg DL, Caiaffa WT, and Correia MI: Hospital malnutrition: the Brazilian national survey (IBRANUTRI): a study of 4000 patients. Nutrition 17(7-8): 573$580,2001$.
Received: December 14, 2010 / Accepted:July 1, 2011

Address for correspondence:

Alexander Karl, MD

Department of Urology

University of Munich-Campus Grosshadern

Marchioninistr. 15

81377 Munich

Germany

Tel.: $\quad+49897095-0$

Fax.: +49897095-8890

E-mail: alexander.karl@med.uni-muenchen.de 